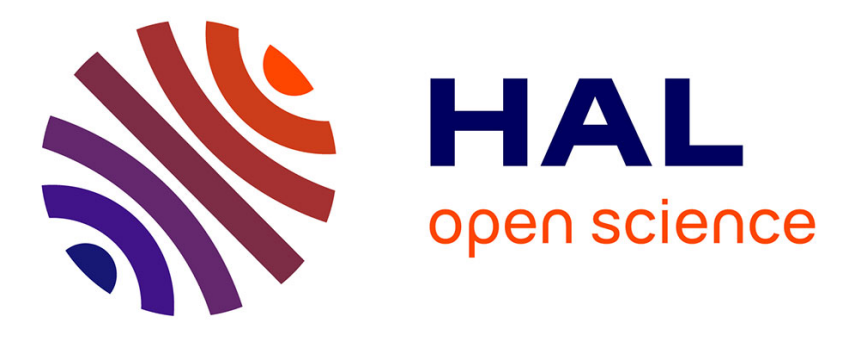

\title{
Human Factors Issues for the Design of a Cobotic System
}

Théo Moulières-Seban, David Bitonneau, Jean-Marc Salotti, Jean-François

Thibault, Bernard Claverie

\section{- To cite this version:}

Théo Moulières-Seban, David Bitonneau, Jean-Marc Salotti, Jean-François Thibault, Bernard Claverie. Human Factors Issues for the Design of a Cobotic System. AHFE 2016 International Conference on Human Factors in Robots and Unmanned Systems, Jul 2016, Orlando, Floride, United States. 10.1007/978-3-319-41959-6_31 . hal-01670381

\section{HAL Id: hal-01670381 https://hal.science/hal-01670381}

Submitted on 21 Dec 2017

HAL is a multi-disciplinary open access archive for the deposit and dissemination of scientific research documents, whether they are published or not. The documents may come from teaching and research institutions in France or abroad, or from public or private research centers.
L'archive ouverte pluridisciplinaire $\mathbf{H A L}$, est destinée au dépôt et à la diffusion de documents scientifiques de niveau recherche, publiés ou non, émanant des établissements d'enseignement et de recherche français ou étrangers, des laboratoires publics ou privés. 


\title{
Human Factors Issues for the Design of a Cobotic System
}

\author{
Théo Moulières-Seban ${ }^{1}$, David Bitonneau ${ }^{1}$, Jean-Marc Salotti ${ }^{2}$, \\ Jean-François Thibault ${ }^{1}$ and Bernard Claverie ${ }^{2}$ \\ 1. Safran SAFRAN, 46 rue Camille Desmoulins, 92130 Issy-les-Moulineaux, \\ France. \\ 2. IMS laboratory, ENSC, 109 Av. Roul, 33400 Talence, France. \\ theo.moulieres-seban@u-bordeaux.fr, david.bitonneau@u-bordeaux.fr, \\ jean-francois.thibault@safran.fr, jean-marc.salotti@ensc.fr, \\ bernard.claverie@ensc.fr
}

\begin{abstract}
We present a new approach for the design of cobotic systems. It is based on several steps with increasing complexity: Activity analysis, basic design, detailed design and realization. A particular attention is paid to human factors and human systems interactions. Different simulation levels are required to provide flexibility and adaptability.
\end{abstract}

Keywords: Human Factors · Cobotics - Ergonomics - Robotics · Cognitics -Workstation design

\section{Introduction}

Cobot is a neologism formed by the "collaborative" and "robot" terms. It has been used for the first time in 1999 by Peshkin and Colgate to conceptualize the direct interaction between a robot and a human on a dedicated workstation [7]. Its meaning evolved towards different definitions depending on the context of the application [4]. In the present study, a cobot is defined as a robot that has been designed and built to collaborate with humans. A workstation in which a robot and a human are collaborating is called a cobotic system. Cobotics is defined by the science and methods of designing, building, studying and evaluating cobotic systems.

A robot may have typical mechanical and hardware components for a possible collaboration with humans but if it is used in full autonomy, it is considered that it is not part of a cobotic system even if it can be called a cobot. Conversely, a standard industrial robot collaborating with an operator (by remote control for instance), is considered the part of a cobotic system.

This paper firstly presents a characterization of cobotic systems, then proposes a methodological approach to introduce cobotic systems on workstations. A use case of a cobotic workstation design at Safran illustrates this approach. 


\section{Cobotic Systems}

Characterization of cobotic systems is very important for industry in order to understand the feasibility, the efficiency and the relevance of designing and implementing a new cobotic system for an industrial application.

A cobotic system includes a robot and a human collaborating in synergy to perform a task in the context of a workstation. In order to characterize a cobotic system, it is necessary to pay attention to the human operator, to the task, to human system interactions and to the robot. Several humans and several robots may be involved in a cobotic system but for the sake of simplicity, we will focus here on a simple cobotic system that involves a unique robot and a unique human operator.

\subsection{Task characterization}

A task is defined by numerous variables [3]. The first one is the domain of application (industrial, domestic, medical, military, etc.). The proposed study is restricted to the industrial domain. Examples of tasks considered in this study are transporting, moving and carrying objects, assembling, surface processing, welding, cutting engraving, etc. The task can also be described by its variability and its necessary adaptation to new applications. Another important variable is the possible impact of a dysfunction or damage on the whole production process [10]. If there is an important risk of failure or a risk to human health, the use of a cobotic system might not be appropriate.

\subsection{Role of Operator}

In the past, only experts in robotics were using robots. Nowadays, more and more people are used to robots and it sometimes happens that newcomers have to interact with industrial robots without training. However, knowledge and knowhow greatly influence our perception and representation of robots, and our understanding of what they can do and what they cannot. It is of primary importance for the industry to design robots that anyone can easily work and interact with after very short training periods. The complexity of the interaction mainly depends on the role of the person at the workstation [8]:

- Operator: He pilots the robot (locally or remotely). The robot usually has a weak autonomy or even no autonomy at all.

- Coworker: He works with the robot on the same object.

- Supervisor: He provides instructions and checks the work of the robot.

- Bystander: He is present in the working zone of the robot without interaction. There is, however, a preliminary risk assessment to make sure that there is no risk with the current task.

- Maintenance operator: He checks and eventually updates mechanical parts, hardware or software components.

- Designer/programmer: Expert in robotics, he designs, builds or develops software tools and advanced behaviors for the robot. 
An important characteristic of the human role concerns the decision process. It can be the result of a common planning, an order, a consensus between the cobot and the human, or an autonomous decision. Parasuraman \& Sheridan propose 10 levels for the decision process, ranging from full assistance to no assistance at all [6].

\subsection{Human System Interactions}

The design of a cobotic system involves a clear understanding of the possible human robot interactions, both needs, both constraints and the type of robotic system [4]. The proximity between the operator and the robot is a crucial parameter for obvious security reasons. Ergonomic reasons must also be taken into account. The robot can be in contact with the operator (comanipulation for instance), nearby, or very far. Sometimes, the robot can be carried by the user (exoskeleton) or the user can be carried by the robot (robotic vehicle) [9]. Interactions may occur in real time with immediate feedback or be differed. In addition, the interaction can be brief, e.g., pushing a button, or continuous (comanipulation). Yanco and Drury propose to characterize the cobotic system by the type of interaction and the type of interface [10]. The sensor used for the interaction has an important impact on the abstraction of the message that is exchanged between the operator and the robot.

The operator can remotely interact with the robot by several means:

- Physically: button, joystick, mouse, handling a robot or end effector replica.

- Using touch-sensitive surfaces: screen or simple touch-sensitive surfaces.

- Visually (information for visual feedback): screen, glasses (virtual or augmented reality), by distance measurements.

- Using motion capture: eyetracking, fingertracking, arm motion tracking, or full body motion tracking.

- Soundly: voice recognition, alarm, oral communication.

In artificial intelligence, computer vision and speech recognition techniques allow high level interactions. However, in industrial applications, the complexity and robustness of these techniques are still considered not appropriate. Object recognition by humans is typically more efficient than computer vision techniques. For that reason, efficient cobotic systems are often made of a robotic manipulator that is directly operated by a person, who is in charge of the perception of the environment.

\subsection{Classification of robots}

The traditional classification of robots is based on their morphology, which usually allows a visual and functional representation of their use:

- Robotic arm: Made of a serial kinematic chain.

- Parallel robot: Robot with ending components linked to the base by several independent kinematic chains.

- Cartesian robot: Robot with prismatic articulations in which axes are located according to Cartesian coordinates.

- Mobile robot: Unmanned vehicles. 
- Exoskeleton: Robot worn by a human to improve its performance or mitigate his handicap.

- Hybrid robot: Combination of the above morphologies.

There are other classification methods [1], [2]. One of them is based on the "intelligence" level of the robot, as it is proposed by the American Robotic Industries Association and the JIRA (Japan Industrial Robot Association). The basic robot is an open loop command system and the most sophisticated is able to elaborate a complex planning process. Another classification has been proposed by Coiffet, see Table 1. It is an interesting approach that takes the environment and humans into account. However, there is no reference to the morphology of the robot.

Table 1. Robots classification.

\begin{tabular}{|c|c|c|c|c|}
\hline Entity & Human & Control system & Robot & Environment \\
\hline \multirow{4}{*}{$\begin{array}{l}\text { Features af- } \\
\text { fecting per- } \\
\text { formances } \\
\text { during the } \\
\text { execution of } \\
\text { task }\end{array}$} & $\begin{array}{l}\text { Continuous } \\
\text { action }\end{array}$ & Open loop & Fixed & Known \\
\hline & $\begin{array}{l}\text { Intermittent } \\
\text { action }\end{array}$ & Regulation & Mobile & Partially known \\
\hline & No action & $\begin{array}{l}\text { Regulation and } \\
\text { reflex }\end{array}$ & & Unknown \\
\hline & & $\begin{array}{l}\text { Regulation, reflex } \\
\text { and decision }\end{array}$ & & \\
\hline
\end{tabular}

\subsection{Scheme to describe a cobotic system}

We propose a characterization scheme. It is based on the information flow among the three components of a cobotic system: the environment of the workstation, the human and the robot. The generic scheme is presented Fig.1.

Interestingly, different cobotic systems have in general different schemes representing the information flow. Fig.2 and Fig.3 are two representative examples of the differences among the cobotic systems:

- For a remotely controlled system, the flow of information between the environment of the task and the operator systematically goes through the robotic system, the operator does not interact directly with the environment ;

- In the last example, an exoskeleton assists the operator without any interaction with the environment.

A given scheme describing an information flow does not always match with a unique type of cobotic system. Another important parameter is the abstraction level of information. If it is simple (data) or complex (object identification by vision for instance) the scheme might be the same but the role of each component might be completely different. A complementary idea is to use different types of links to provide the abstraction level. 


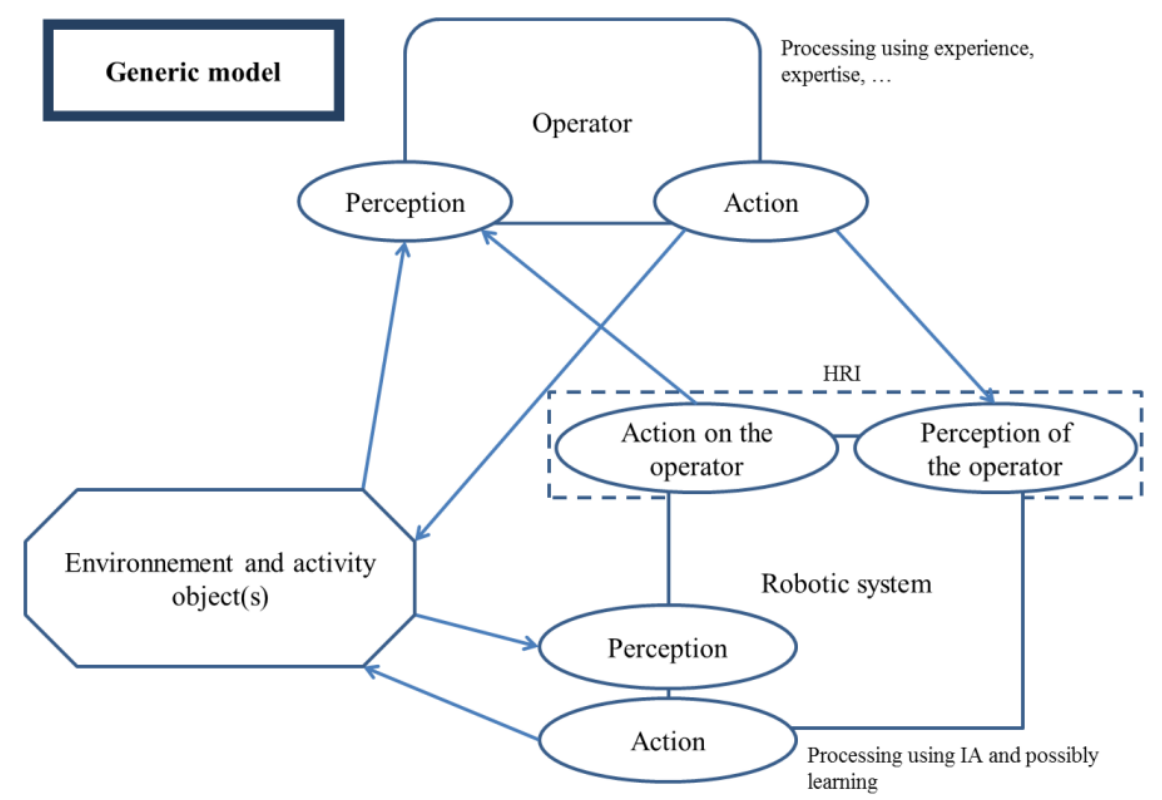

Fig. 1. Standard scheme of a cobotic system.

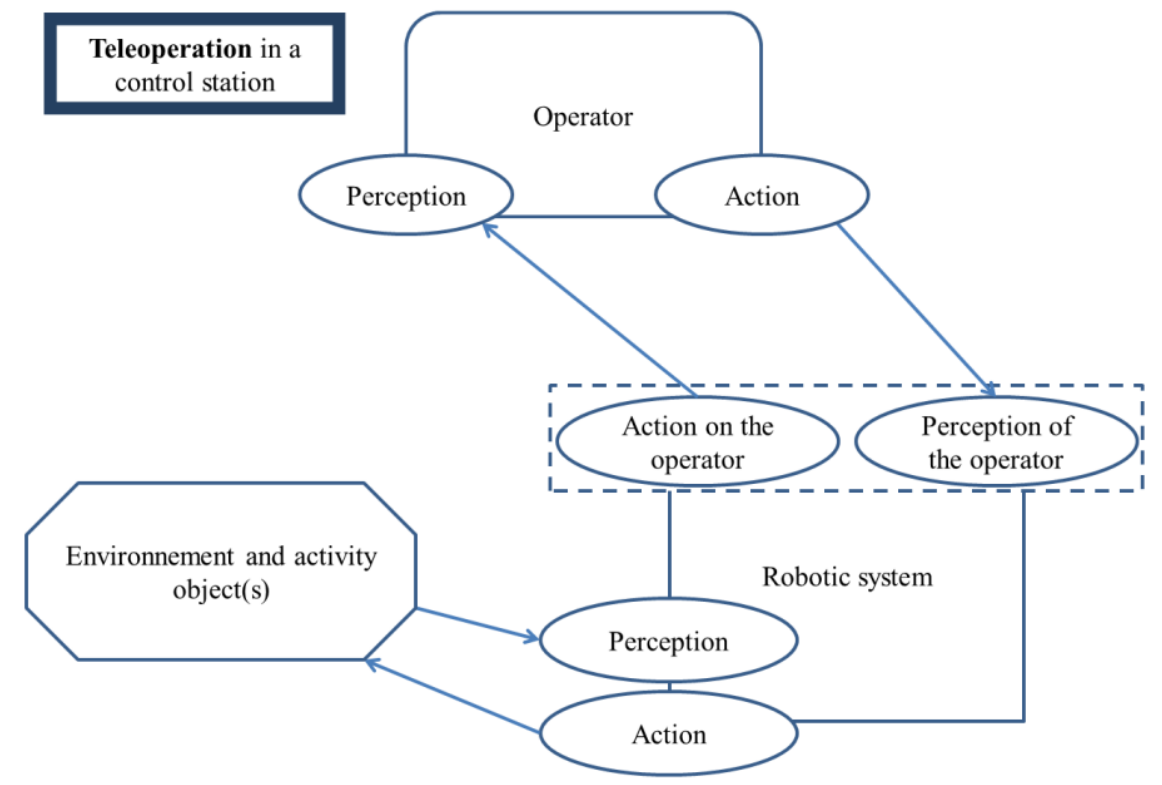

Fig. 2. Scheme of a teleoperating system. 


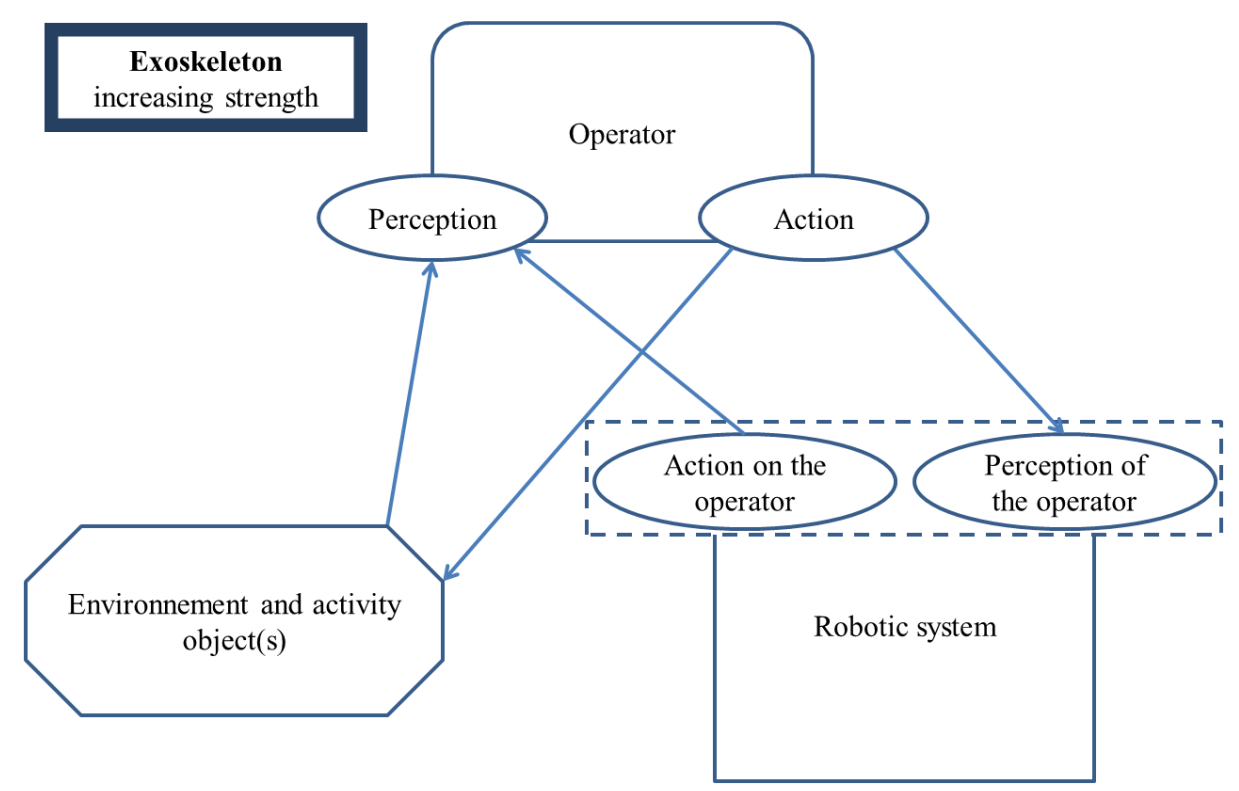

Fig. 3. Scheme of an exoskeleton system.

\section{Practical Case and Methodological Approach}

A dedicated human centered design approach is proposed to determine the functional specifications of a cobotic system. The method is currently implemented within a Safran cobotic project (tank cleaning) with the collaboration of researchers from the Cognitics and Human Engineering team of the IMS laboratory and Ecole Nationale Supérieure de Cognitique. The previous cobotic systems' characterization is a valuable tool that enables a classification of all cobotic solutions in order to match the requirements of the workstation.

Let us consider an application. Nowadays, cleaning viscous and sticky chemical product off huge propellant tanks is carried out manually. An operator scrubs the tank using simple tools (kinds of spade). For a long time, operating at this workstation has been an issue because it is hard, tiresome and performed in a hazardous environment. As the task is long, complex and variable, a full automation is considered very difficult. The current objective is to design a cobotic system for that task. The idea is to minimize the presence of the operator at the station to reduce operational risks and improve working conditions to preserve operator's health.

\subsection{Task, environment and context analysis}

The first step is the analysis of the current activity: the task, the environment and the context. A preliminary work is the study of other similar projects eventually with existing solutions. 
The main work consists in interviewing the operators, their manager, anyone that is involved in the project. Then, it is crucial to observe the accomplishment of the task itself. The objective of this analysis is to explicit how the task is really performed, and the reason why it is performed this way to understand the workstation stakes. As many variables (concerning the product, the environment, the tools, the communication among the operators, etc.) as possible have to be identified. In order to assist the operator with a cobotic system, it is important to identify his skills and experience, and the phases for which he has no or few expertise.

The output of this step is a document including the detailed functional specifications of the system. This document allows the first exchanges with experts in automation, the proposal of possible solutions (only the basic principles) and possible suppliers. If the technology readiness level (TRL) of the proposed solution is too low, the feasibility has to be checked by means of technical tests.

\section{Example: Safran's cobotic project}

The cleaning tank analysis led to the different products and their state, the different tank's dirtiness, and the different techniques of the operator to clean the tank. Two solution's principles were considered : robotic scrubbing and hydrogomming. The tests revealed that the hydrogomming, which had a low TRL, was a heavy going process, inappropriate to the shape of the tank. The robotic scrubbing has been chosen. Testing it permitted to decide its size and strength.

\subsection{Basic design}

Here, the solution is to design and realize scenarios and mockups. At first, they cannot be accurate, but once implemented, they can be corrected and improved again and again, until they are validated by everyone involved in the project.

The mockup should present the best tradeoff between the time it takes to develop it and update possible solutions, and the distance to the industrial process. Virtual reality tools can be used [5]. Two benefits are expected with the mockup: it allows anticipating errors by testing design hypothesis, and it helps the operators, the designers and the decision-makers to share the same representation of the future workstation. The second point is important because it improves the acceptability of the system. An operator may indeed be afraid of losing his job.

The outputs of the basic design are the specifications for a prototype.

The mockup also allows increasing the TRL of the proposed systems and subsystems to level 5 or 6 .

\section{Example: Safran's cobotic project}

In the cleaning tank project, we realized an interactive mockup of a teleoperation system. We simulated a workstation with three camera views and a data screen. Along the half-automated cycle, we could test different ways to interact with the simulated robot: a joystick, a haptic device, etc (Fig. 4). Several operators tried the simulator and we are currently validating the mockup. 

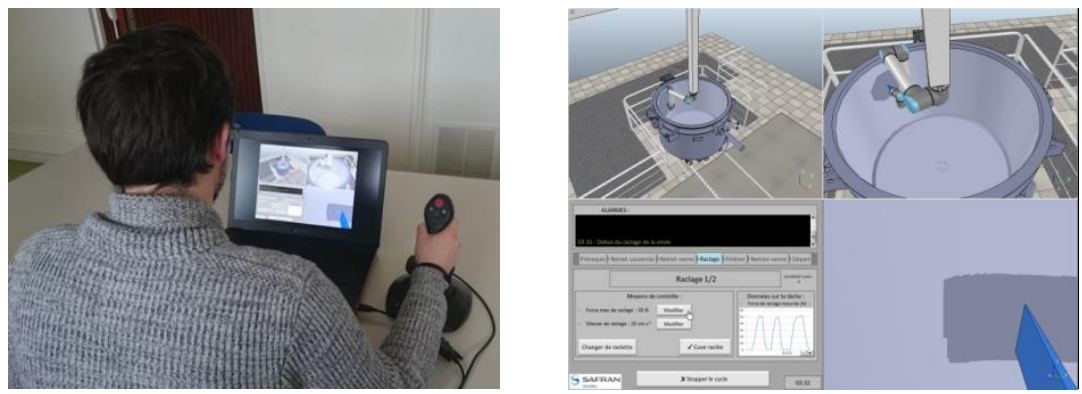

Fig. 4. Picture and screenshot of the mockup realized for the cleaning tank project.

\subsection{Detailed design}

The detailed design is the logic next step after the basic design: the principle is to design the solution using a prototype (temporary version of the final system). A first imprecise design is done, then tested. After that, corrections and improvements can be done and tested again, etc., until everyone involved in the project validates it. Thus, the operators can try their future way of performing their task, and help improving it. Simulating these interactions is decisive when designing a cobotic system.

The TRL increases to level 7 or 8 .

The outputs of this step are the technical specifications for the final system, coming to a solicitation of offers from suppliers.

\section{Example: Safran's cobotic project}

When the base design is finished, we plan to realize a prototype at supplier's test facility and experiment with it. The results of the experiments will lead us to the technical specifications of the cobotic system.

\subsection{Production and adjustment}

Once the suppliers have answered the solicitation, at this step, the best one is selected according to several criterions (quality of the solution, cost, experience, etc.), and realizes the cobotic system, followed up by the project team. Then, the supplier and possibly the concerned department of the enterprise, install, adjust and validate it before it is put in service. The project team has to make sure that the cobotic workstation is adapted to all the operators and the production cycle, and that the operators are trained enough to work with the system. 
Table 2. Overview of the methodological approach.

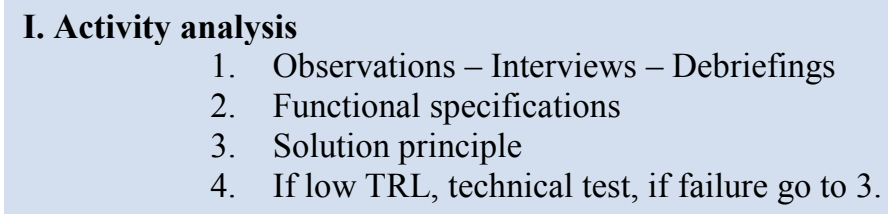

\section{Basic design}

1. Exchange with experts

2. Design and realization of the mockup

3. Test with the mockup, if corrections or enhancement needed go to 2.

4. Validation and specifications for a prototype

\section{Detailed design}

1. Choice of a supplier response for the prototype

2. Design and realization of the prototype

3. Test the prototype, if corrections or enhancement needed go to 2 .

4. Validation and technical specifications for the final cobotic system

\section{Realization, setup, validation and putting into service}

\section{Conclusion}

The proposed methodological approach has to be carried out and achieved with the current use case and then tested on other use cases to validate, correct and complete it.

This approach is gobal and can be adapted to most cobotic situations. A specific emphasis is placed on the analysis of human / robot interactions. Different elements have to be considered depending on the exact interaction scheme. Several experts from three different disciplines are involved in the project: ergonomics for the analysis of the workstation the variability of the tasks, cognitive engineering to design the human robot interactions and robotics for the robot itself. This multidisciplinary aspect is a source of wealth for the project.

\section{References}

1. Alami, R., Chatila, S., Fleury, M., Ghallab, M., \& Ingrand, F.. An architecture for autonomy. International Journal of Robotics Research, 17, p. 315-337, (1998).

2. Angeles, J., \& Park, F. C.. Performance Evaluation and Design Criteria. In B. \&. Siciliano, Springer Handbook of Robotics (pp. 229-244). Secaucus, NJ, USA: Springer-Verlag New York, Inc. (2007).

3. Barcellini, F., Van Belleghem, L., \& Daniellou, F. Design projects as opportunities for the development of activities. In P. Falzon, Constructive ergonomics (pp. 150-163). Boca Raton, FL, USA: CRC Press, (2014). 
4. Goodrich, M. A., \& Schultz, A. C.. Human-Robot Interaction : A Survey. Foundation and Trends ${ }^{\circledR}$ in Human-Computer Interaction, Vol. 1, No. 3, p. 203-275, (2007).

5. Maurice, P., Virtual Ergonomics for the design of collaborative robots (Thèse de doctorat). Université Pierre et Marie Curie, Paris IV, (2015).

6. Parasuraman, R., \& Sheridan, T. B., A Model for Types and Levels of Human Interaction with Automation. IEEE Transactions on Systems, Man, and Cybernetics - Part A: Systems and Humans, vol. 30 (3), (2000).

7. Peshkin, M., \& Colgate, J., Cobots. Industrial Robot: An International Journal Volume 26 . Number 5 . 1999 . pp. 335-341, (1999).

8. Scholtz, J., Theory and Evaluation of Human Robot Interactions. Proceedings of the $36^{\text {th }}$ Hawaii International Conference on System Sciences (HICSS'03), (2003).

9. Walther, S., \& Guhl, T., Classification of physical human-robot interaction scenarios to identify relevant requirements. Conference ISR Robotik, (2014).

10. Yanco, H. A., \& Drury, J., Classifying Human-Robot Interaction : An Updated Taxonomy, (2004). 